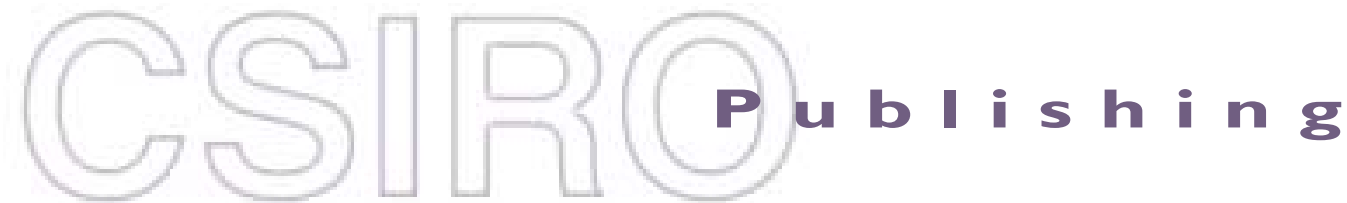

\section{Publications of the Astronomical Society of Australia}

Volume 19, 2002

(C) Astronomical Society of Australia 2002

An international journal of astronomy and astrophysics

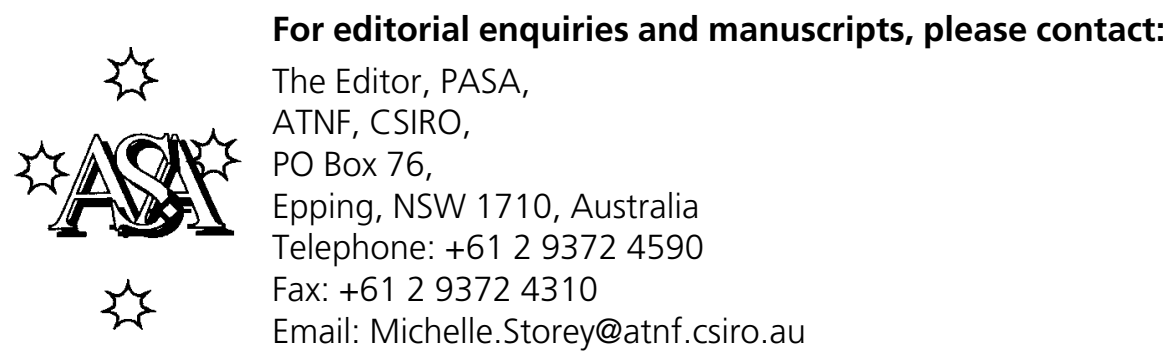

For general enquiries and subscriptions, please contact: CSIRO Publishing PO Box 1139 (150 Oxford St)

Collingwood, Vic. 3066, Australia

Telephone: +6139662 7666

Fax: +61 396627555

Email: publishing.pasa@csiro.au

C S I RO

PUBLISHING Published by CSIRO Publishing

for the Astronomical Society of Australia

www.publish.csiro.au/journals/pasa 


\title{
Optical to Far-IR Observations of 3C 446
}

\author{
K. J. Leech ${ }^{1}$, R. Saxton ${ }^{2}$, P. Barr ${ }^{3}$, M. Santos-Lleo ${ }^{4}$, E. Jiminez-Bailon ${ }^{5}$, \\ R. Diaz ${ }^{6}$, K. Ghosh ${ }^{7}$ and S. Soundararajaperumal ${ }^{7}$ \\ ${ }^{1}$ ISO Data Centre, Astrophysics Division, ESA Space Science Department, \\ P.O. Box 50727, 28080 Madrid, Spain \\ Kieron.Leech@said-business-school.oxford.ac.uk \\ ${ }^{2}$ Vega/ESA, XMM-SOC, University of Leicester, University Road, \\ Leicester LE1 7RH, UK \\ rds@star.le.ac.uk \\ ${ }^{3}$ Astrophysics Division, ESA Space Science Department, ESTEC Postbus 299, \\ 2200 AG - Noordwijk, The Netherlands \\ Paul.Barr@astro.estec.esa.nl \\ ${ }^{4}$ XMM Science Operations, Astrophysics Division, ESA Space Science Department, \\ P.O. Box 50727, 28080 Madrid, Spain \\ msantos@xmm.vilspa.esa.es \\ ${ }^{5}$ Laeff, P.O. Box 50727, 28080 Madrid, Spain \\ ${ }^{6}$ Observatorio Astronomico de Cordoba/SeCyT, UNC, Argentina \\ ${ }^{7}$ Indian Institute of Astrophysics, Bangalore, 560034, India \\ Received 2001 July 28, accepted 2002 May 24
}

\begin{abstract}
The OVV blazar 3C 446 was monitored over seven months in 1997 with ground based telescopes and instruments on ESA's Infrared Space Observatory (ISO). The aim was to try to detect variations in the IR and see if these were correlated with optical variations. The object varied in the optical and near-IR during this period, but did not vary in the far-IR. Despite being a factor of ten weaker than in 1983, the optical-IR SED exhibited the same slope. The new far-IR observations from ISO allow us to determine the location of the turnover in the spectrum, caused by synchrotron self-absorption. It occurs just longwards of $100 \mu \mathrm{m}$.
\end{abstract}

Keywords: BL Lacertae objects: individual (3C 446) — infrared: galaxies

\section{Introduction}

3C 446 is an OVV blazar at $z=1.4$ with a $L_{\mathrm{FIR}}>10^{13} \mathrm{~L}_{\odot}$. It exhibits rapid variability in the optical, X-ray (e.g. see Sambruna 1997), and radio (e.g. see Terasranta et al. 1998). As an OVV it can exhibit large amplitude optical flux variations on a variety of timescales. Between 1970 and 1986 it varied in $B$ by $\approx 4$ magnitudes (Webb et al. 1988), and between 1986 and 1992 the B variations were of the order of 2 magnitudes (Netzer et al. 1996). In 1997 (when the observations reported here were made) its optical emission was a factor of ten weaker than in 1983, and it exhibited weak optical variations over the time period of a week in June 1997 (Ghosh et al. 2000).

The motivation for this project was to observe blazars to see if they varied in the mid and far-IR. 3C 446 was chosen as a suitable target because we felt its rapid and large variability in the optical gave us a high chance of success in detecting IR flux variations. Subsidiary questions we wanted answering included: Were any IR flux variations correlated with optical variations? Does any of the IR emission originate in a thermal source? To answer these questions we intended to observe 3C 446 from the optical to the far-IR with differing delays between epochs, ranging from hours to months, to find the characteristic variability timescale in the mid and far-IR.

\section{Observations}

A monitoring program was organised with the Infrared Space Observatory (ISO) of the European Space Agency (ESA) (Kessler et al. 1996; see also www.iso.vilspa.esa.es), the $2.34 \mathrm{~m}$ telescope of the Vainu Bappu Observatory, Kavalur, India, and the $1.54 \mathrm{~m}$ telescope of the Observatorio Bosque Alegre, Universidad Nacional de Cordoba, Argentina. Table 1 gives the observing log.

On ISO we used the camera (ISOCAM, Cesarsky et al. 1996) and photometer (ISOPHOT, Lemke et al. 1996) to observe through five filters centred on 6.7, 14.3, 25, 60, and $170 \mu \mathrm{m}$. Near-simultaneous optical observations were made in the $\mathrm{B}, \mathrm{V}, \mathrm{R}$, and I bands from the ground based observatories using $\mathrm{CCD}$ cameras.

The 6.7 and $14.3 \mu \mathrm{m}$ observations were made with the ISOCAM camera, a $32 \times 32$ imaging camera. Each set of two observations lasted 550 seconds.

Each $25 \mu \mathrm{m}$ observation lasted 1400 seconds and was made with the ISOPHOT photometer. This had a $52^{\prime \prime}$ diameter aperture and observed the target in a raster chop pattern of on and off-target pointings.

The 60 and $170 \mu \mathrm{m}$ observations were made with the ISOPHOT cameras $(3 \times 3$ and $2 \times 2$ pixels respectively). Observations were made in a $3 \times 3$ raster pattern with the instrument moving approximately the size of a pixel 
Table 1. Observing log

\begin{tabular}{|c|c|c|c|c|}
\hline Date & $\begin{array}{c}\text { Start } \\
\text { time/UT }\end{array}$ & Where & $\begin{array}{c}\text { Wavelengths } \\
\mu \mathrm{m}\end{array}$ & $\begin{array}{l}\text { TDT numbers for } \\
\text { ISO observations only }\end{array}$ \\
\hline 4-May-1997 & 02:57:09 & ISO & 6.714 .32560170 & 53500204535002055350024053500206 \\
\hline 4-May-1997 & $03: 51: 25$ & ISO & 6.714 .32560170 & 53500307535003085350034153500309 \\
\hline 4-May-1997 & $04: 45: 41$ & ISO & 6.714 .32560170 & 53500410535004115350044253500412 \\
\hline 4-Мay-1997 & 08:54:57 & ISO & 6.714 .32560170 & 53501513535015145350154353501515 \\
\hline 4-Мay-1997 & 17:00:04 & ISO & 6.714 .32560170 & 53501513535028175350284453502818 \\
\hline 5-Мay-1997 & 08:30:53 & ISO & 6.714 .32560170 & 53600319536003205360034553600321 \\
\hline 5-May-1997 & & Argentina & V, R, I & \\
\hline 6-May-1997 & $16: 21: 30$ & ISO & 6.714 .32560170 & 53702822537028235370284653702824 \\
\hline 6-May-1997 & & Argentina & $\mathrm{R}, \mathrm{I}$ & \\
\hline 9-May-1997 & 03:15:31 & ISO & 6.714 .32560170 & 54000325540003475400032754000326 \\
\hline 14-May-1997 & $04: 57: 29$ & ISO & 6.714 .32560170 & 54500428545004305450042954500448 \\
\hline 24-May-1997 & 10:19:42 & ISO & 6.714 .32560170 & 55501031555010325550104955501033 \\
\hline 4-Jun-1997 & & Argentina & $\mathrm{V}, \mathrm{R}, \mathrm{I}$ & \\
\hline 7-Jun-1997 & & India & $\mathrm{V}$ & \\
\hline 8-Jun-1997 & & India & $\mathrm{V}$ & \\
\hline 13-Jun-1997 & 10:11:37 & ISO & 6.714 .32560170 & 57501134575011355750115057501136 \\
\hline 15-Jun-1997 & & India & $\mathrm{V}$ & \\
\hline 13-Jul-1997 & & Argentina & $\mathrm{V}, \mathrm{R}, \mathrm{I}$ & \\
\hline 12-Aug-1997 & & Argentina & $\mathrm{B}, \mathrm{V}, \mathrm{R}, \mathrm{I}$ & \\
\hline 2-Nov-1997 & $15: 40: 32$ & ISO & 6.714 .32560170 & 71800137718001387180015171800139 \\
\hline 16-Nov-1997 & $17: 12: 19$ & ISO & 2560100135170200 & 73200593732005927320059073200691 \\
\hline 20-Nov-1997 & & Argentina & $\mathrm{B}, \mathrm{V}, \mathrm{R}, \mathrm{I}$ & \\
\hline
\end{tabular}

( $46^{\prime \prime}$ and $\left.92^{\prime \prime}\right)$ between each pointing. These observations lasted 600 and 450 seconds each.

Other observations from the ISO archive, not part of our monitoring program, were also used.

\section{Data Reduction}

For the optical data, standard data processing techniques were applied using the standard calibrations, biases, and flat fields available at the two telescopes. The photometric analysis was carried out using Midas and the SEXTRACTOR program on sky-subtracted images. The flux of 3C 446 was measured relative to the flux of a nearby bright star present in the images, and tied to an absolute calibration with photometric standards observed at Bosque Alegre during one of the nights (20 November 1997). The accuracy of the absolute fluxes is to better than 0.15 magnitudes.

For the ISOCAM data standard pipeline products were processed with CIA (ISOCAM Interactive Analysis, Ott et al. 1997), and synthetic aperture photometry (with a 6 " aperture) was used in extracting the fluxes from the images.

For the $25 \mu \mathrm{m}$ ISOPHOT data standard pipeline products were processed and the fluxes extracted using PIA (PHOT Interactive Analysis, Gabriel et al. 1997).

For the 60 and $170 \mu \mathrm{m}$ ISOPHOT data standard pipeline products were processed with PIA. Individual detector drifts were removed and detector-to-detector variations flat fielded out. All pixels whose centres lay within 10" $\left(40^{\prime \prime}\right.$ for the $170 \mu \mathrm{m}$ data) of the target position were considered on-source and the average of off-source pixels (defined to be more than $70^{\prime \prime}$ or $100^{\prime \prime}$ away) was subtracted.

\section{Results and Conclusions}

3C 446 was in a low state during 1997, with flux a factor of 10 lower than when observed by IRAS in 1983. Nevertheless, it did vary in the optical and near-IR during the observing period, as can be seen from Figures 1 and 2 . The optical minimum was around early June 1997 (June 1 is JD-2450601), with a halving time of approximately 10 days in the rest frame. The 7,14 , and $25 \mu \mathrm{m}$ observations also showed a minimum around this period, with a similar halving time. The far-IR (60 and $170 \mu \mathrm{m})$ observations did not, however, show any variations.

There was no change in the optical-IR spectral index of approximately -1.2 between 1983 and 1997, as can be seen from Figure 3. This is despite the drop in flux by a factor of 10 . Neither does there seem to be a difference in the spectral index between the high and low states of 1997.

As the wavelength coverage of the ISO instruments extends to over $200 \mu \mathrm{m}$, compared to $100 \mu \mathrm{m}$ for IRAS, the new data allows us to locate the turnover in the power law, caused by self-absorption. This lies between $100 \mu \mathrm{m}$ and $170 \mu \mathrm{m}$. While this may be considered a very high frequency for self-absorption, for example if we use formula 3.12 from Verschuur \& Kellermann (1988) it implies the magnetic field is of the order of $10^{15} \mathrm{G}$, the models have many free parameters. The alternative explanation that the emission is due to cold gas at $20-30 \mathrm{~K}$ has the problem that the host galaxy would then become an ultraluminous FIR galaxy, with large amounts of dust which 


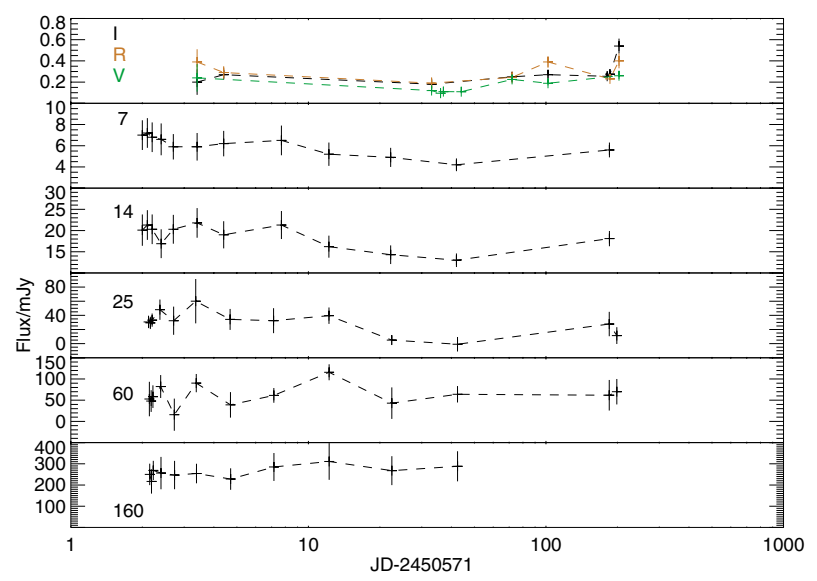

Figure 11997 variability of 3C 446 in log time.

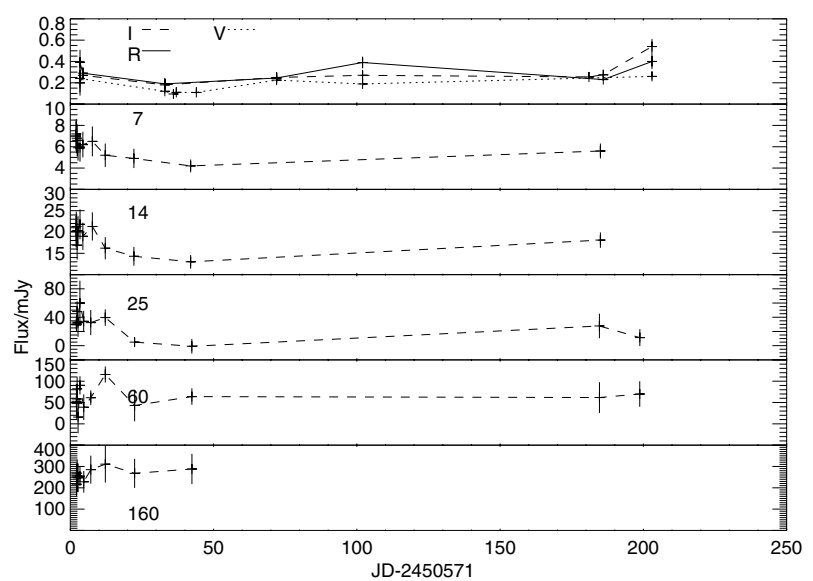

Figure 21997 variability of 3C 446 in linear time.

would cause extreme reddening of the optical/UV nucleus. No evidence for such reddening is seen, and therefore there is no evidence that any of the IR emission is thermal.

\section{Acknowledgments}

Based on observations with ISO, an ESA project with instruments funded by ESA Member States (especially the PI countries: France, Germany, the Netherlands and the United Kingdom) and with the participation of ISAS and NASA.

The ISOPHOT data presented in this paper were reduced using PIA, which is a joint development by the ESA Astrophysics Division and the ISOPHOT

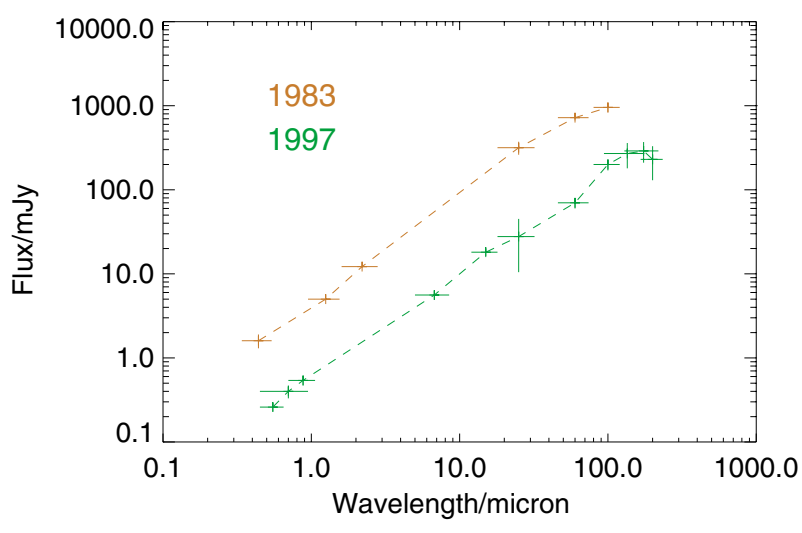

Figure 3 The 1983 and November 1997 SEDs of 3C 446. The horizontal bars indicate the bandpass of the filters, the vertical bars the error estimate. The November 1997 ISO data is from 2 November 1997, the optical data from 20 November 1997.

Consortium with the collaboration of the Infrared Processing and Analysis Center (IPAC). Contributing ISOPHOT Consortium institutes are DIAS, RAL, AIP, MPIK, and MPIA.

The ISOCAM data presented in this paper were analysed using 'CIA', a joint development by the ESA Astrophysics Division and the ISOCAM Consortium. The ISOCAM Consortium is led by the ISOCAM PI, C. Cesarsky.

\section{References}

Cesarsky, C. J., et al. 1996, A\&A, 315, L32

Gabriel, C., Acosta-Pulido, J., Heinrichsen, I., Morris, H., \& Tai, W.-M. 1997, in Astronomical Data Analysis Software and Systems (ADASS) VI, ASP Conference Series 125, eds G. Hunt, \& H. E. Payne (San Francisco: ASP), 108

Ghosh, K. K., Ramsey, B. D., Sadun, A. C., \& Soundararajaperumal, S. 2000, ApJS, 127, 11

Kessler, M. F., et al. 1996, A\&A, 315, L27

Lemke, D., et al. 1996, A\&A, 315, L64

Netzer, H., et al. 1996, MNRAS, 279, 429

Ott, S., et al. 1997, in Astronomical Data Analysis Software and Systems (ADASS) VI, ASP Conference Series 125, eds G. Hunt, \& H. E. Payne (San Francisco: ASP), 34

Sambruna, R. M. 1997, ApJ, 487, 536

Teraesranta, H., et al. 1998, A\&AS, 132, 305

Verschuur, G. L., \& Kellermann, K. I., eds. 1988, Galactic and Extragalactic Radio Astronomy, Springer-Verlag

Webb, J. R., Smith, A. G., Leacock, R. J., Fitzgibbons, G. L., Gombola, P. P., \& Shepherd, D. W. 1988, AJ, 95, 374 